\title{
Possible roles of electrical synapse in temporal information processing: a computational study
}

\author{
Xu-Long Wang, Xiao-Dong Jiang \& Pei-Ji Liang * \\ Department of BiomedicalEngineering, Shanghai Jiao TongUniversity. *Correspondence should be addressed to Pei-Ji Liang(pjliang@sjtu.edu.cn).
}

\begin{abstract}
Temporal information processing in the range of tens to hundreds of milliseconds is critical in many forms of sensory and motor tasks. However, little has been known about the neural mechanisms of temporal information processing. Experimental observations indicate that sensory neurons of the nervous system do not show selective response to temporal properties of external stimuli. On the other hand, temporal selective neurons in the cortex have been reported in many species. Thus, processes which realize the temporal-to-spatial transformation of neuronal activities might be required for temporal information processing. In the present study, we propose a computational model to explore possible roles of electrical synapses in processing the duration of external stimuli. Firstly, we construct a small-scale network with neurons interconnected by electrical synapses in addition to chemical synapses. Basic properties of this small-scale neural network in processing duration information are analyzed. Secondly, a large-scale neural network which is more biologically realistic is further explored. Our results suggest that neural networks with electrical synapses functioning together with chemical synapses can effectively work for the temporal-to-spatial transformation of neuronal activities, and the spatially distributed sequential neural activities can potentially represent temporal information.
\end{abstract}

Keywords: Model; Neural network; Electrical synapse; Temporal information processing

\section{INTRODUCTION}

Biological neural systems are endowed with the ability to process temporal information given the inherent temporal nature of sensory environments and motor tasks. Neuroscientists roughly categorize temporal information processing in the neural system into four different time scales: microseconds, milliseconds, seconds and circadian rhythm, which serve for different physiological functions and rely on different neural mechanisms. The process within the scale of millisecond is perhaps the most sophisticated and the least well understood one among these categories. Behavioral tasks with temporal information processing falling within this scale include speech discrimination in the auditory system, motion information processing in the visual systems, and movement coordination in the motor system [1-3].

Information processing in neural systems normally consists of a number of successive stages. Neural activities in a certain stage are mostly determined by neural activities of the preceding stages and our perception of the world in the brain is based on the spatio-temporal patterns of neuronal activities produced at sensory stages [4-5]. Physiological observations indicate that neurons in the sensory levels do not respond selectively to the temporal properties of external stimuli. Temporal information is thus suggested to be contained in the temporal patterns of neuronal activities in the sensory layer. On the other hand, neurons which show selective response to specific temporal properties, especially the duration content, have been reported in the cortex of many species [6-10]. Temporal information is therefore suggested to be transformed into the spatially distributed neuronal activities in the cortex and neural mechanisms which contribute to the temporalto-spatial transformation of neuronal activities are required.

Electrical synapse is another type of widely distributed neuronal connection in the neural systems in addition to chemical synapse [11-12]. Functional role of electrical synapse has been identified in fine motor coordination which requires temporal information processing in milliseconds scale [13]. In the present work, we try to explore possible neural mechanisms of electrical synapse in processing the duration content of external stimuli via computational approach. Briefly, we construct neural net- 
works containing both electrical and chemical synapses, which are activated by stimuli with various durations. Computational results show that electrical synapse can substantially contribute to the temporalto-spatial transformation of neuronal activities, and the neuronal activities in such networks can potentially represent information about stimulus durations.

\section{MODELS AND METHODS}

\subsection{Model structure}

Two types of computational models are constructed. One is a small-scale neural network which contains only several tens of neurons. Another is a large-scale one which is more biologically realistic. We use the simple model to clarify the basic properties of neural networks with electrical synapses functioning together with chemical synapse in temporal information processing. The overall behavior is further tested in the large-scale model which is more biologically realistic.

The schematic structures of the small- and largescale neural networks are illustrated in Figure 1, A and $B$ respectively. Stimuli with various durations are applied, as represented by various durations of the input currents. The input current is injected to an input neuron (S) and then transformed into spike train s of this neu ron.

The input neuron is connected to some of the ten excitatory neurons (E) in the small-scale model. Electrical synapses are presented among assigned neurons, as indicated in the figure. Excitatory neurons are connected to each other recurrently by chemical synapses and each excitatory neuron is further coupled with an inhibitory neuron (I) to ensure its stability. Parameters used in the small- scale model are listed as follows:

$I S_{i p}$ : Intensity of the input current;

$C S_{s e}$ : Strength of chemical synapse from input neuron to excitatory neurons;

$C S_{e e}$ : Strength of chemical synapse between excitatory neurons;

$E S_{e e}:$ Strength of electrical synapse between excitatory neurons;

$C S_{e i}$ : Strength of chemical synapse from excitatory neurons to inhibitory neurons;

$C S_{i e}$ : Strength of chemical synapse from inhibitory neurons to excitatory neurons.

The large-scale neural network model contains 400 excitatory neurons and 100 inhibitory neurons. The ratio between the excitatory and inhibitory neurons follows the experimental observations from neocortical area [14]. The neural network is further divided into 100 subgroups with each subgroup consisting of 4 excitatory neurons and 1 inhibitory neuron. Excitatory and inhibitory neurons in each individual subgroup are connected recurrently. Input neuron is connected to excitatory and inhibitory neurons on a random basis. All excitatory neurons are further connected with each other probabilistically in a recurrent way, and the synaptic strengths are variables which follow normal distributions. Parameters used for synaptic connections in the extended model are listed as follows:

$C P_{\text {se }}$ : Probability of chemical synapse from input to excitatory neurons;

$C M_{s e}$ and $C D_{s e}$ : Mean and standard deviation of strength of chemical synapse from input to excitatory neurons;

$C P_{s i}$ : Probability of chemical synapse from input

B

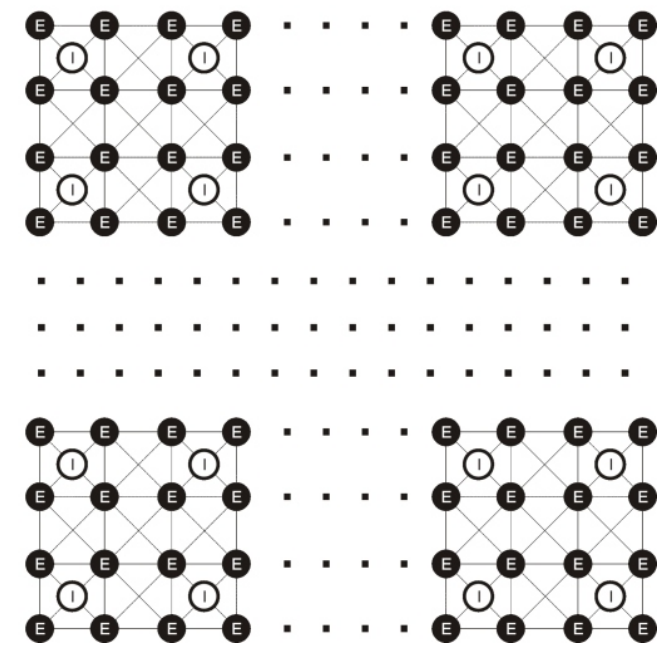

Figure 1. A. Schematic structure of the small-scale neural network model. The input neuron (S) is connected to 4 of the 10 the excitatory neurons $(E)$. All excitatory neurons are connected to each other in a recurrent way and each excitatory neuron is coupled with an inhibitory neuron (I). Excitatory and inhibitory synapses are represented by open and solid circles, respectively. Neurons in grey shadow are electrically coupled together recurrently.B. Schematic structure of the large-scale neural network model. Input neuron is connected to excitatory (E) and inhibitory (I) neurons in the network on a random basis. All excitatory neurons are further connected with each other probabilistically in a recurrent way. Electrical synapses are formed between some of the excitatory neurons randomly. 
to inhibitory neurons;

$C M_{s i}$ and $C D_{s i}$ : Mean and standard deviation of strength of chemical synapse from input to inhibitory neurons;

$C P_{e e}$ : Probability of chemical synapse between excitatory neurons;

$C M_{e e}$ and $C D_{e e}$ : Mean and standard deviation of strength of chemical synapse between excitatory neurons;

$C M_{e i}$ and $C D_{e i}$ : Mean and standard deviation of strength of chemical synapse from excitatory to inhibitory neurons;

$C M_{i e}$ and $C D_{i e}$ : Mean and standard deviation of strength of chemical synapse from inhibitory to excitatory neurons;

$E P_{e e 1}$ : Probability of electrical connection between excitatory neurons within one subgroup;

$E P_{e e 2}$ : Probability of electrical connection between excitatory neurons in different subgroups;

$E M_{e e}$ and $E D_{e e}$ : Mean and standard deviation of strength of electrical synapse between excitatory neurons.

\subsection{Mathematical description of neurons and} synapses

\subsubsection{Description of integrate-and-fire neuron}

Neurons are described in an integrate-and-fire manner (I-F neuron) [5]. Membrane potential of the input neuron $\left(V_{s}\right)$, excitatory neuron $\left(V_{E x}\right)$, and inhibitory neuron $\left(V_{I n}\right)$ can be determined as follows:

$$
\begin{aligned}
C \cdot \frac{d V_{S}}{d t}= & g_{\text {leak }} \cdot\left(V_{e q}-V_{S}\right)+I_{S} \\
C \cdot \frac{d V_{E x}}{d t}= & g_{\text {leak }} \cdot\left(V_{e q}-V_{E x}\right)+\left[g_{e x}(t) \cdot\left(E_{e x}-V_{E x}\right)\right. \\
& \left.+g_{\text {in }}(t) \cdot\left(E_{\text {in }}-V_{E x}\right)\right]+I_{\text {esyn }} \\
C \cdot \frac{d V_{\text {In }}}{d t}= & g_{\text {leak }} \cdot\left(V_{\text {eq }}-V_{\text {In }}\right)+\left[g_{\text {ex }}(t) \cdot\left(E_{e x}-V_{\text {In }}\right)\right. \\
& \left.+g_{\text {in }}(t) \cdot\left(E_{\text {in }}-V_{\text {In }}\right)\right]
\end{aligned}
$$

where

$C$ represents the membrane capacitance;

$V_{e q}$ denotes the equilibrium membrane potential;

$g_{\text {leak }}$ is the leak conductance;

$g_{e x}$ and $g_{\text {in }}$ represent the conductance of excitatory and inhibitory synapses, respectively;

$E_{e x}$ and $E_{i n}$ represent the reversal membrane

potentials of excitatory and inhibitory synapses, respectively;

$I_{\text {esyn }}$ represents the current passing through electrical synapses.

In addition, when the membrane potential reaches a threshold $\left(V_{t h}\right)$, the neuron fires an action potential, and the membrane potential is immediately reset to the equilibrium potential $\left(V_{e q}\right)$ after a firing lasting time $\left(T_{\text {fire }}\right)$.

Parameter values chosen for the I-F neuron model are listed in Table 1 . These values are mostly adopted from Troyer and Miller (1997) [15], except that the firing lasting time of inhibitory neurons is chosen as 4 to ensure the neurons' inhibitory effect on the activities of excitatory neurons.

\subsubsection{Description of synaptic current}

The chemical synapses are modeled as follows [1617]:

$$
I_{c s y n}=g_{c s y n} \cdot g(t) \cdot\left(E-V_{\text {post }}\right)
$$

where $g_{e x}(t)$ and $g_{i n}(t)$ in eqns (2) and (3) are presented by $g_{\text {csyn }}(t) \cdot g(t)$ here, with $g_{\text {csyn }}$ representing synaptic strength which is modified by a factor of $g(t)$ :

$$
\frac{d g(t)}{d t}=\frac{1}{\tau_{s y n}} \cdot[f(t)-g(t)]
$$

where

$$
\frac{d f(t)}{d t}=\frac{1}{\tau_{s y n}} \cdot\left[\Theta\left[V_{p r e}-E_{t h r}\right]-f(t)\right]
$$

in which $\tau_{\text {syn }}=15 \mathrm{~ms}, \mathrm{E}_{t h r}=-40 \mathrm{mV}$, and $\Theta(u)$ follows a step function:

$$
\begin{cases}\Theta(u)=0 & u \leq 0 \\ \Theta(u)=1 & u>0\end{cases}
$$

The electrical synapses are described as follows:

$$
I_{\text {esyn }}=g_{\text {esyn }} \cdot\left(V_{\text {pre }}-V_{\text {post }}\right)
$$

Where $g_{\text {csyn }}$ represents the synaptic strength. We adopt this abstract function which simply depicts that the current passing through the electrical synapses is generally dependent on the membrane potential difference between the pre-synaptic and postsynaptic neurons [18].

\section{RESULTS}

Table 1. Parameter values for the I-F neuron model. The firing lasting time $\left(T_{\text {fire }}\right)$ for sensory and excitatory neurons is set as $1.75 \mathrm{~ms}$ whereas that for inhibitory neuron is set as $4 \mathrm{~ms}$.

\begin{tabular}{ccccccc}
\hline $\mathrm{C}$ & $\mathrm{V}_{\text {eq }}$ & $V_{\text {th }}$ & $g_{\text {leak }}$ & $E_{\text {ex }}$ & $E_{\text {in }}$ & $T_{\text {fire }}$ \\
$(p F)$ & $(m V)$ & $(m V)$ & $(\mu S)$ & $(m V)$ & $m V)$ & $(m s)$ \\
\hline 0.5 & -74 & -54 & 0.025 & 0 & -74 & $1.75 / 4$ \\
\hline
\end{tabular}


A
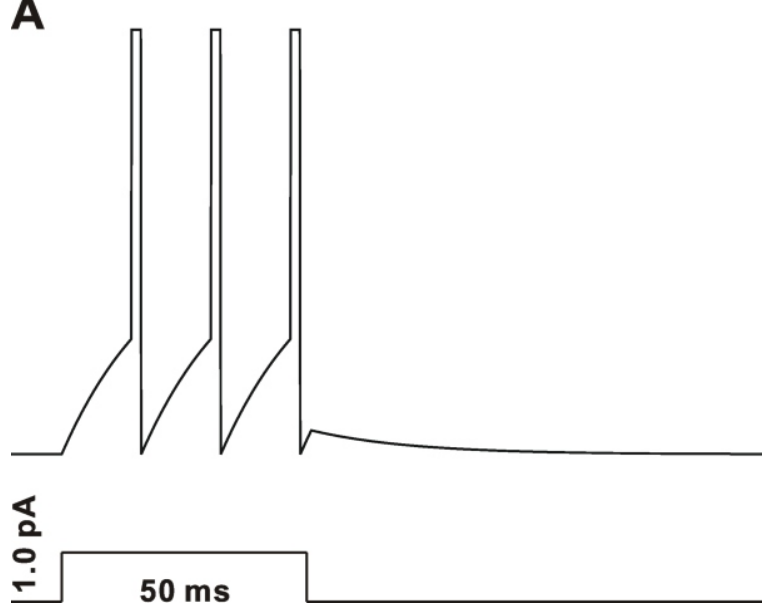

C
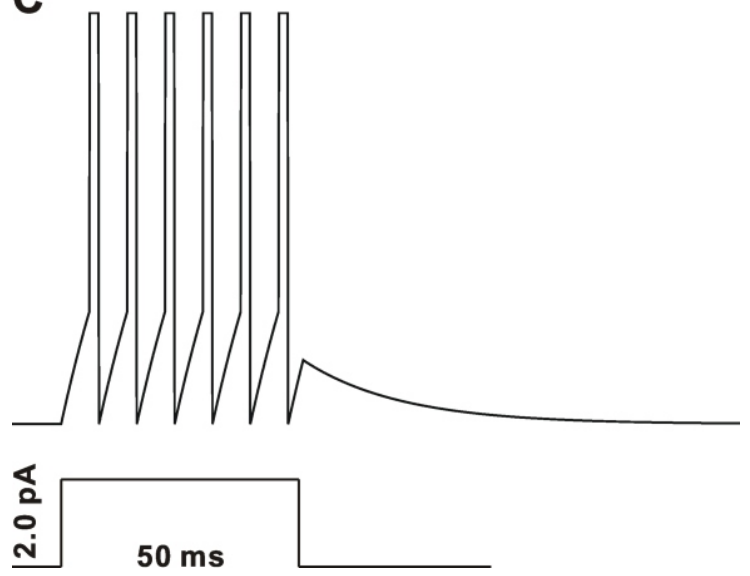

B

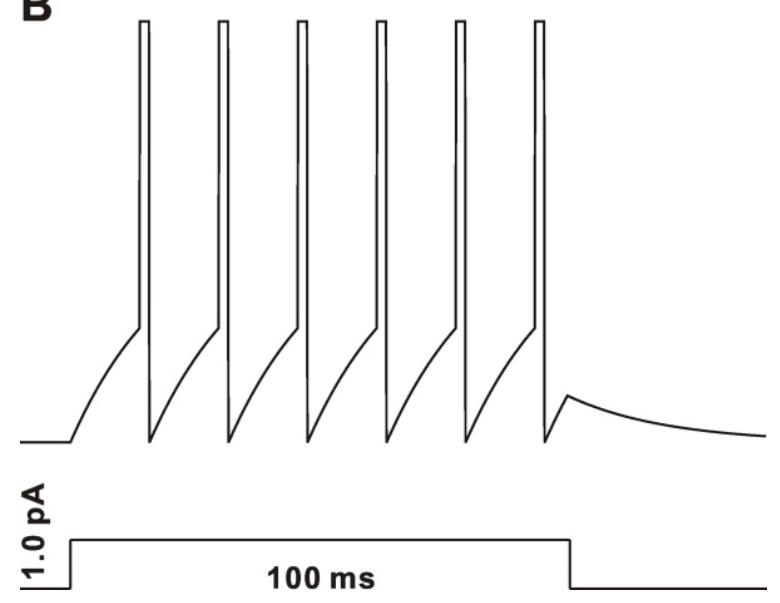

D

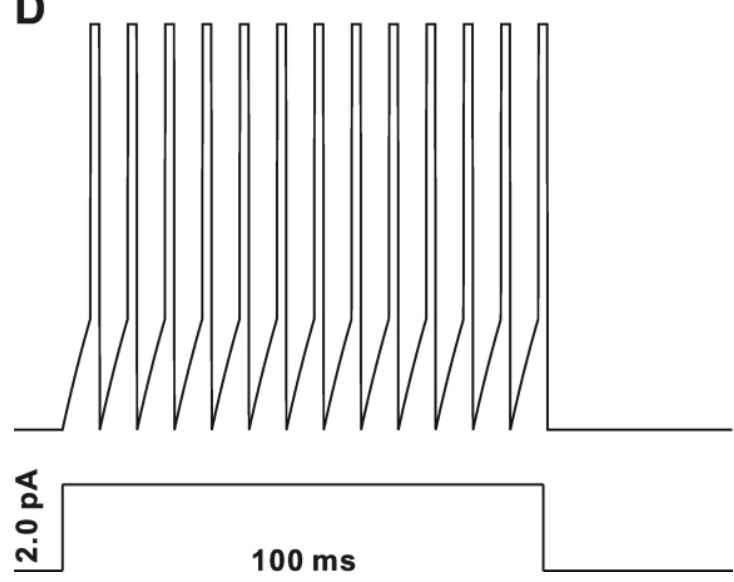

Figure 2. Spike activities of the input neuron $(S)$ in response to constant injected currents with various intensities and magnitudes.

3.1 Stimulus duration is represented by spike trains of input neuron

The injected current is first transformed into a spike train of the input neuron. Spiking properties of the input neuron (S) are shown in Figure 2. Injected currents with different magnitudes and durations are applied to the input neuron to test its performance. A sustained current elicits periodic spikes from the input neuron and the duration of the spike train is determined by the stimulus duration. Input neuron can therefore mimic the function of sensory neuron in neural system.

\subsection{Performance of the small-scale neural network model}

3.2.1 Temporal information can be represented by the spatially distributed activities of a group of neurons

Representative firing patterns of the simple model are given in Figure 3. Parameters used for Figure 3 are listed in Table 2 and the synaptic connection follows that illustrated in Figure 1A. Input neuron is connected to four of the ten excitatory neurons. Three neuronal groups are electrically coupled together which contain 2, 3 and 4 neurons, respectively. Raster plots of the firing performances of the model neurons in absence and presence of electrical synapses are compared with stimulus duration being $50 \mathrm{~ms}$ (Figure $\mathbf{3 A} \& \mathbf{B}$ ) and $100 \mathrm{~ms}$ (Figure 3C\&D), respectively.

Results given in Figure 3 B \&D suggest that electrical synapses in a neural network can effectively transform the temporal domain spike train of the input neuron into the spatial-temporal firing pattern of a group of neurons. Each activated neuron in the group fires within a specific time window, which is determined by the configuration of the synaptic connection of the neural network. Fur therm ore, stimulus with longer duration can evoke spikes from more neurons and therefore the stimulus durations can be represented by the spatial and temporal structure of the sequential neuronal activities.

\subsubsection{The output pattern is closely related to the electrical coupling configuration}

Electrical synapses between excitatory neurons and 
A

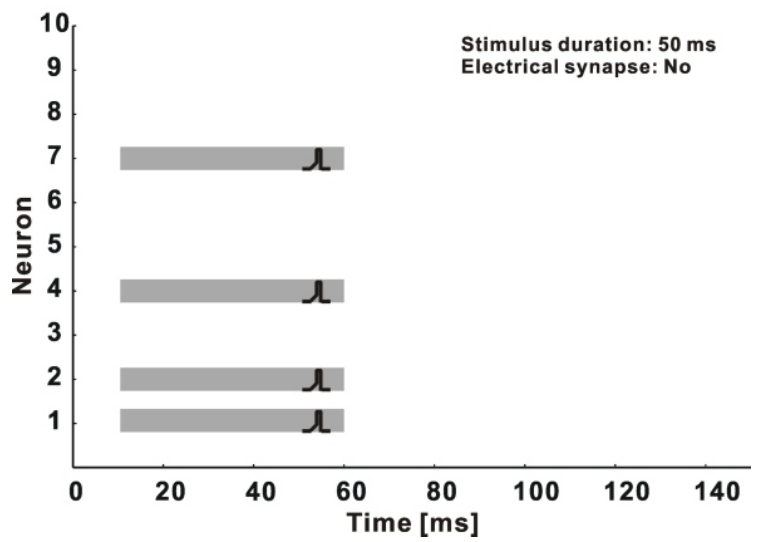

C

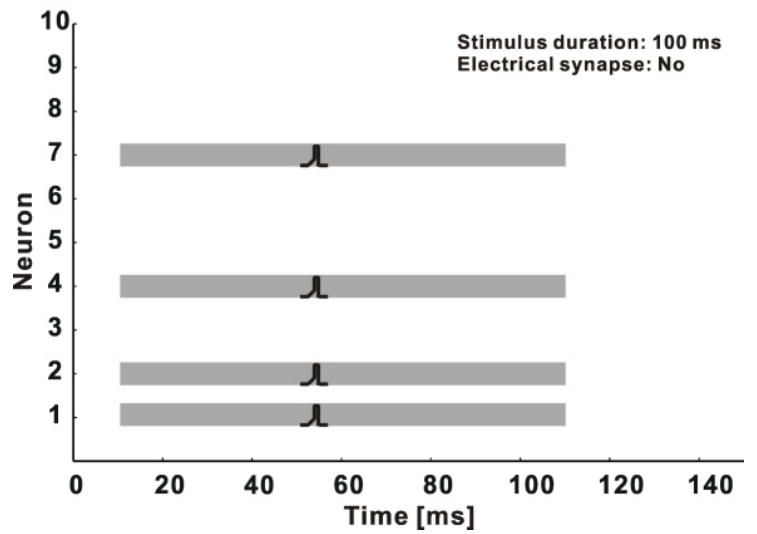

B

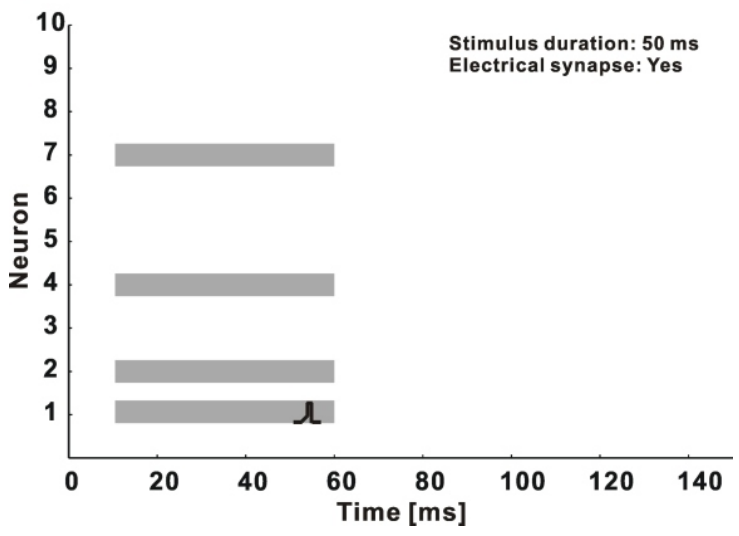

D

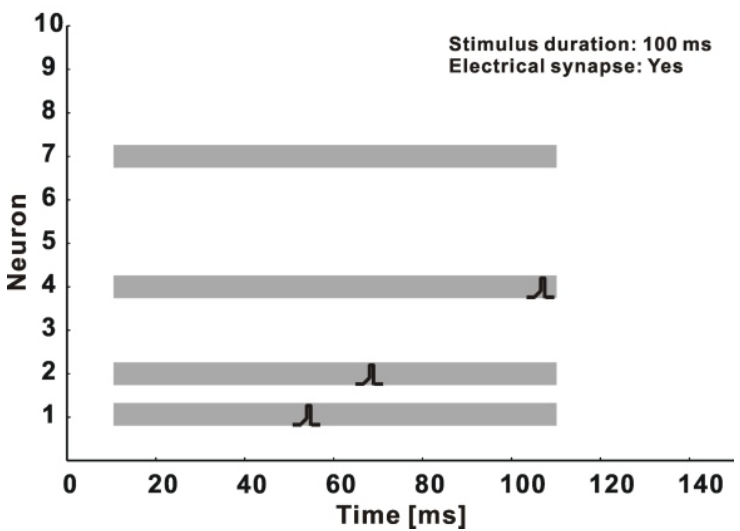

Figure 3. Raster plots for neuronal activities of the small-scale model elicited by 50 and $100 \mathrm{~ms}$ stimulus durations. Stimuli are indicated by grey shadows. A, 50 ms duration, without electrical synapses; B, 50 ms duration, with electrical synapses; C, $100 \mathrm{~ms}$ duration, without electrical synapses; D, $100 \mathrm{~ms}$, with electrical synapses.

Table 2. Parameter values used in the small-scale neural network model.

\begin{tabular}{cccccc}
\hline$I S_{i p}(p A)$ & $C S_{s e}(\mu S)$ & $C S_{e e}(\mu S)$ & $E S_{e e}(\mu S)$ & $C S_{e i}(\mu S)$ & $C S_{i e}(\mu S)$ \\
\hline 2.0 & 0.075 & 0.0001 & 0.02 & 1.0 & 2.0 \\
\hline
\end{tabular}

synaptic connections from input neuron to the network are important factors that influence the model's performance. There are three groups of neurons electrically coupled together in the smallscale model presented in Figure 1A. Neurons within each group are all electrically coupled in a recurrent manner. Furthermore, only one neuron in each group is connected to the input neuron. The model outputs in response to stimuli with different durations are presented in Figure 3. However, any change in the configurations of the electrical coupling and input neuron connection may also cause relevant changes in the results. Take the 3-neuron group in Figure $1 \mathrm{~A}$ (E4, E5 and E6) for an example, relevant possibilities of the electrical coupling within this group as well as the chemical synapses between these neurons and the input neuron are tested, with the rest structure of the neural network kept unaltered. The spiking activities of these three neurons under the test conditions are plotted in Figure 4. The firing activities are quite different with different synap tic config urations. Generally, spikes can be elicited from the neurons that are chemically connected to the input neuron, and longer delay is produced when the chemically activated neuron is electrically coupled with more neurons that do not receive chemical input from the input neuron (e.g. A\&F vs B\&D).

\subsection{Performance of the large-scale neural network model}

Performance of the small-scale model suggests a mechanism for temporal information processing in a neural network containing electrical synapses. In real neural network, the synaptic strengths as well as the 
A

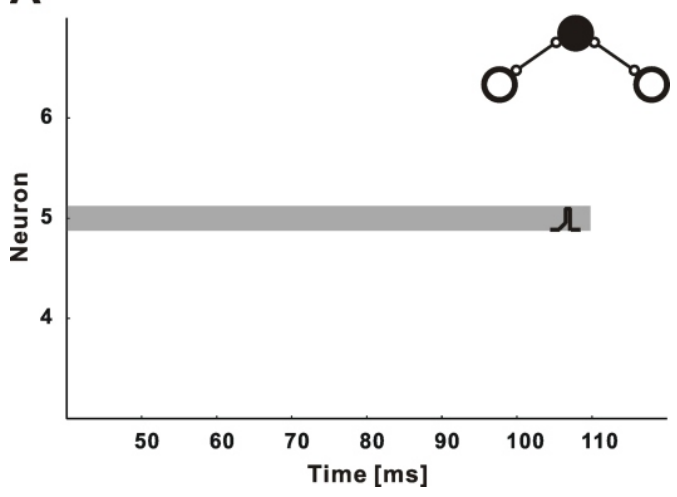

C

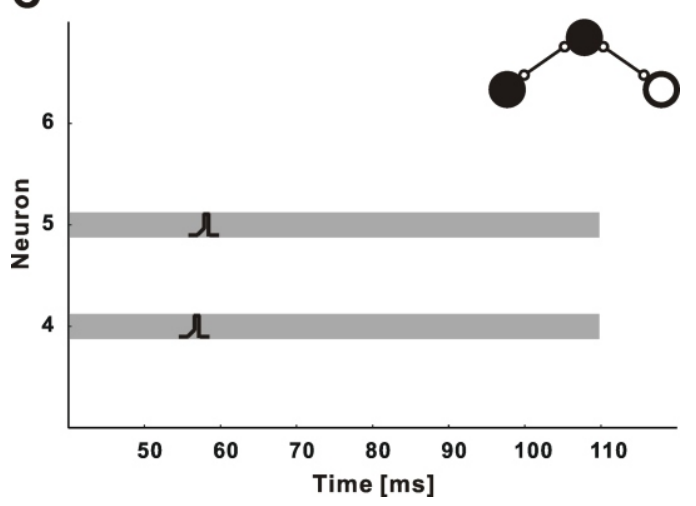

E

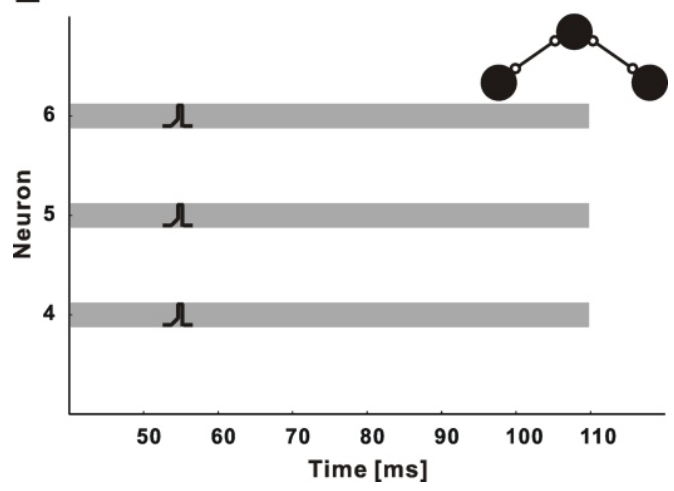

G

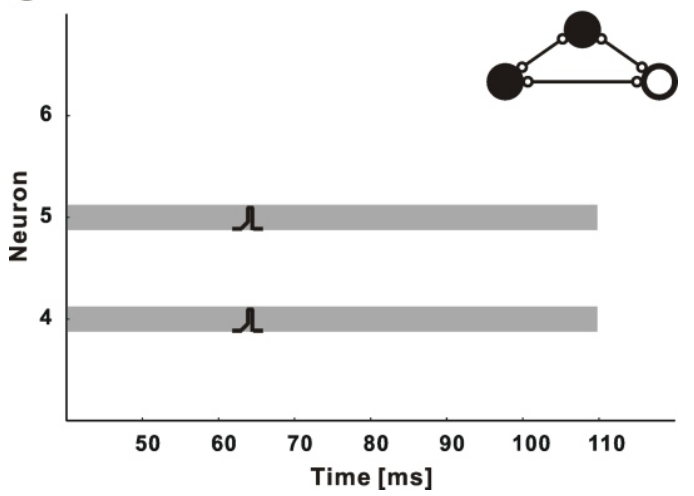

B

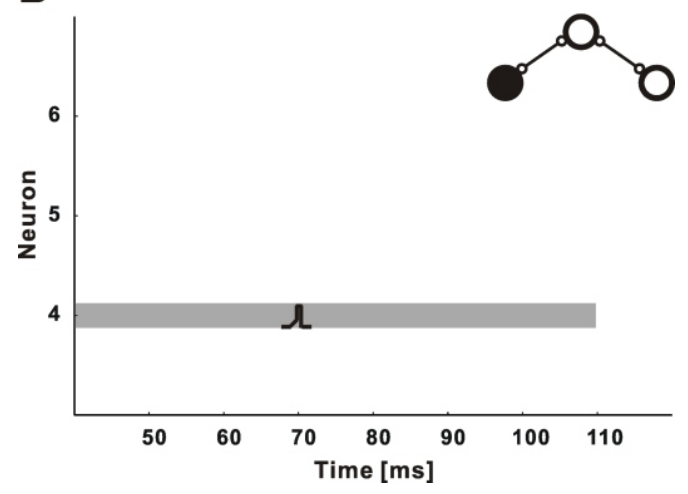

D

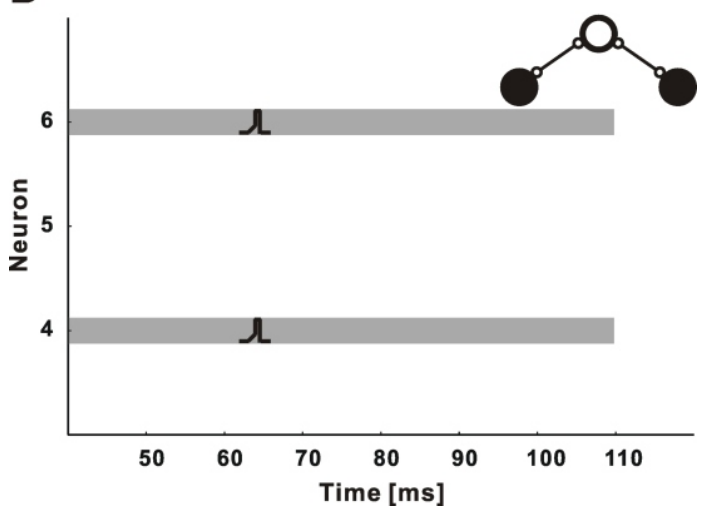

F

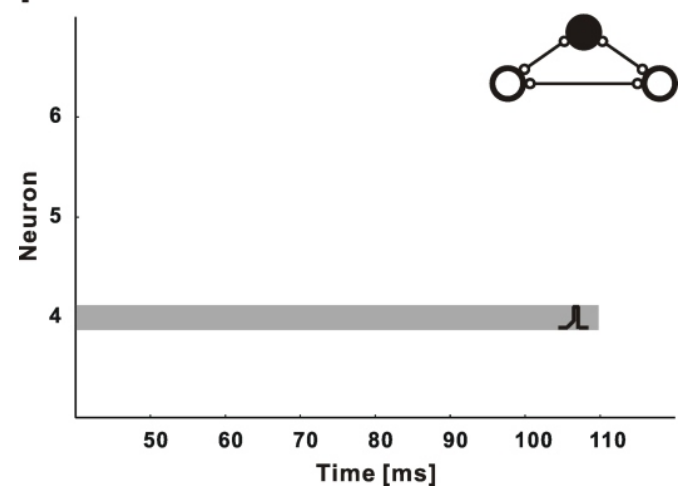

H

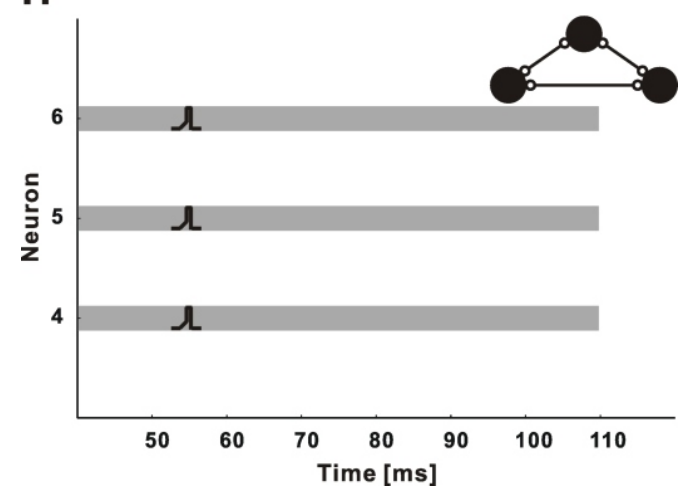

Figure 4. Raster plots for spike activities of threeneuron group with different synaptic configurations. Neurons receive synaptic input from input neuron are represented by solid circle. Electrical synapses are represented by solid lines. The stimulus duration is $100 \mathrm{~ms}$ with the currentintensity to input neuron being $2.0 \mathrm{pA}$. 
A

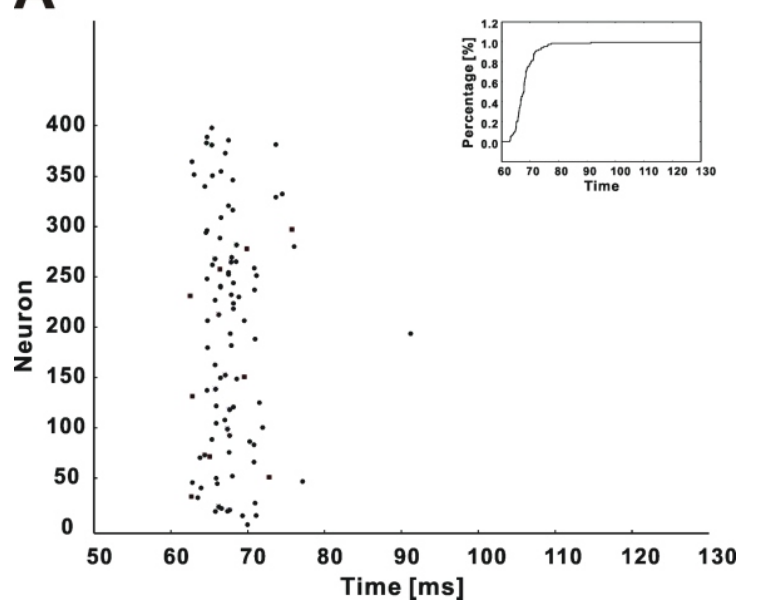

C

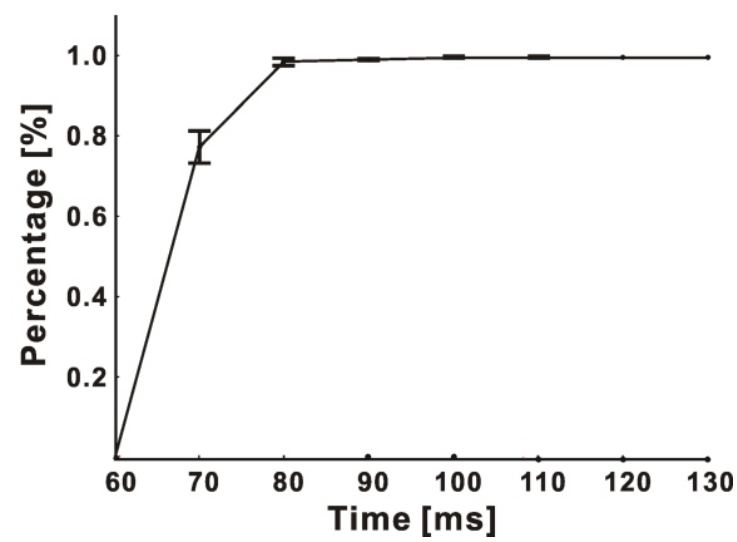

B

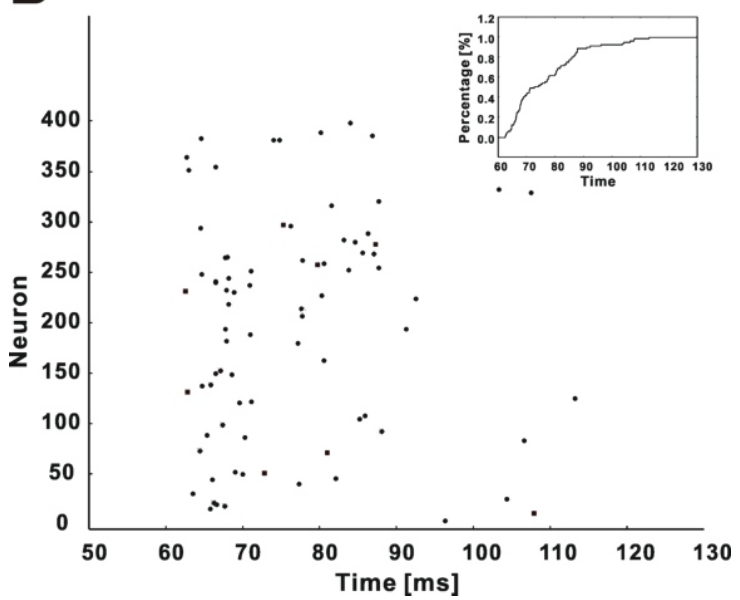

D

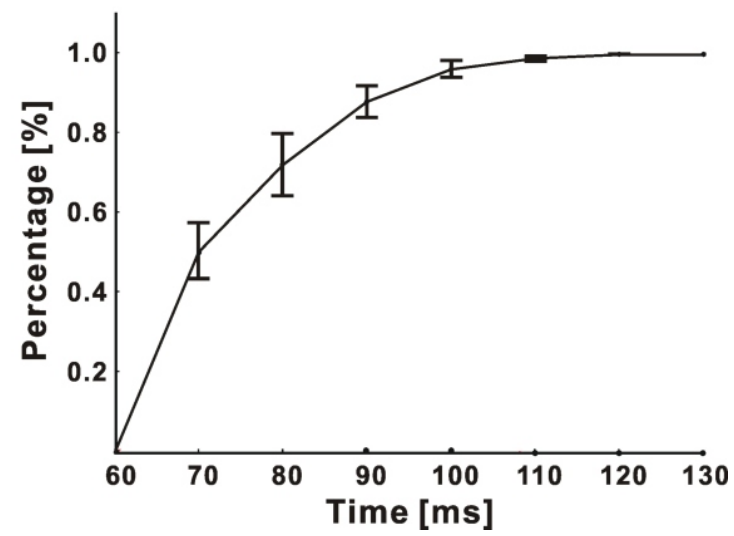

Figure 5. A and B are representative raster plots of the neuronal activities of the large-scale model in absence and presence of electrical synapses, respectively. The stimulus duration is $100 \mathrm{~ms}$. Inset graphs represent the processes of spike activity recruitment. $C$ and $D$ show the recruitment processes in absence and presence of electrical synapses, respectively. Data are averaged based on 10 independent trails (Mean \pm S.D.).

Table 3. Parameter values used in the large-scale neural network model.

$\begin{array}{llll}C P_{s e} & 0.25 & C M_{s e} / C D_{s e}(\mu S) & 0.055 / 0.003 \\ C P_{s i} & C M_{s i} / C D_{s i}(\mu S) & 0.03 / 0.01 \\ C P_{e e} & 0.98 & C M_{e e} / C D_{e e}(\mu S) & 0.001 / 0.001 \\ / & 1 & C M_{e i} / C D_{e i}(\mu S) & 0.2 / 0.01 \\ / & / & C M_{i e} / C D_{i e}(\mu S) & 0.7 / 0.01 \\ E P_{e e 1} & E M_{e e} / E D_{e e}(\mu S) & 0.01 / 0.001 \\ E P_{e e 2} & 0.25 & E M_{e e} / E D_{e e}(\mu S) & 0.01 / 0.001\end{array}$

electrical coupling configuration are not fixed but variable. A large-scale model which is more biologically realistic is constructed with parameter variations, and its performance is tested.

Representative firing patterns of the large-scale model in absence and presence of electrical synapses are shown in Figure $\mathbf{5} \mathbf{A}$ and $\mathbf{B}$, respectively. The stimulus duration time is $100 \mathrm{~ms}$. Neural network parameters used for Figure $\mathbf{5}$ are listed in Table 3.
The inset graphs represent the recruitment process of the neuronal spiking activities. The temporal distribution of the neuronal activities under these two conditions is compared by analyzing the recruitment process in ten independent trials. The results are shown in Figure $\mathbf{5 C}$ and $\mathbf{D}$. It is clear that the presence of electrical synapses results in a broader temporal distribution of the sequential spike activities of the neurons (B \& D), while the neuronal firing 
A

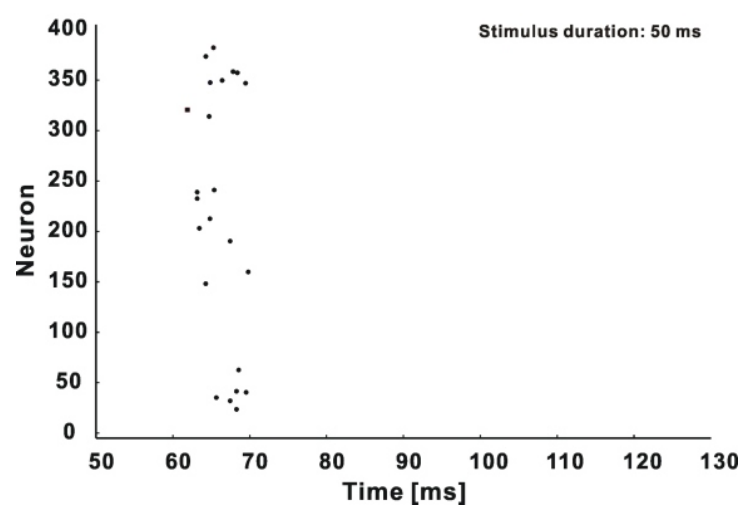

C

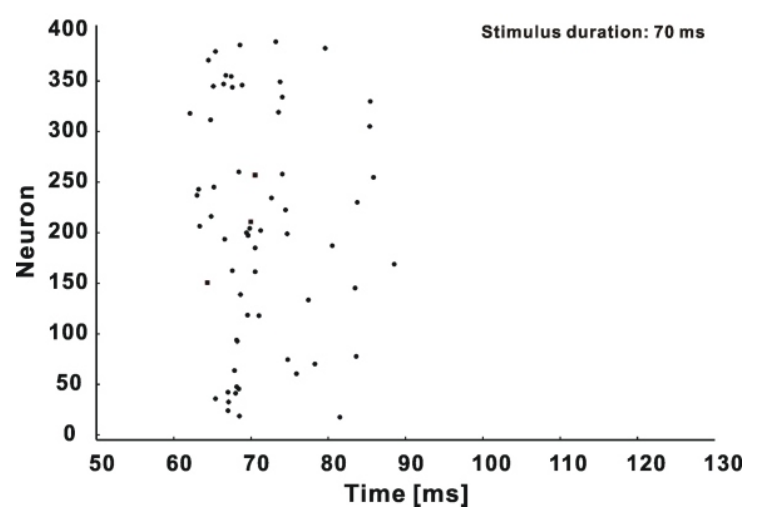

E

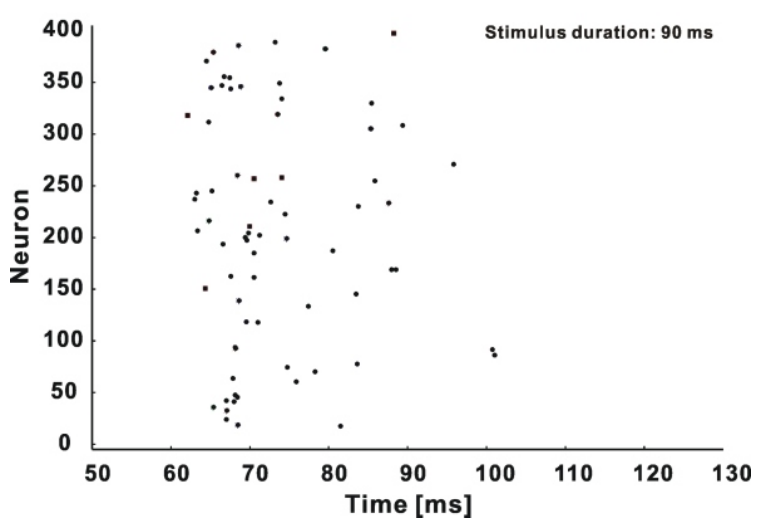

B

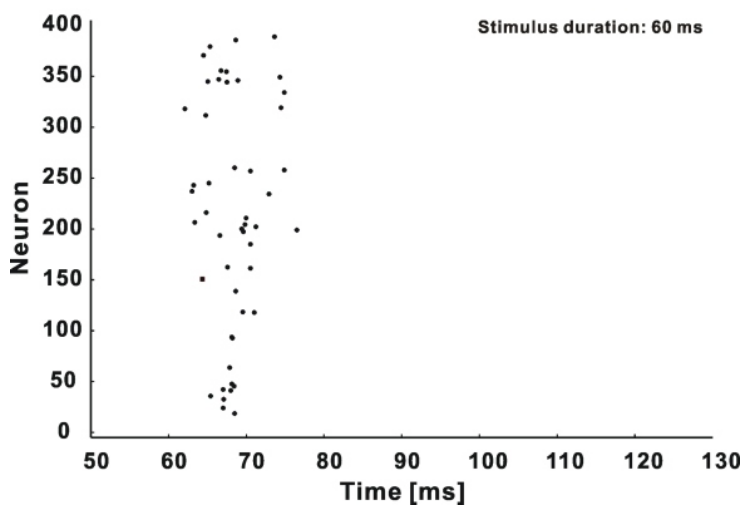

D

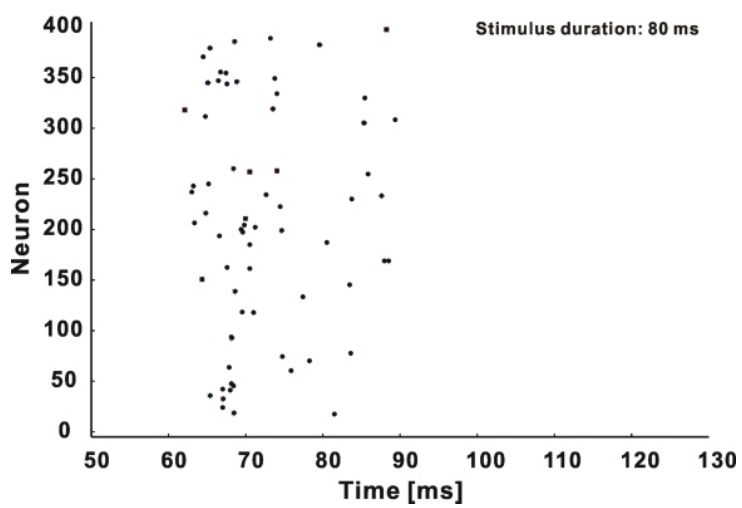

$\mathbf{F}$

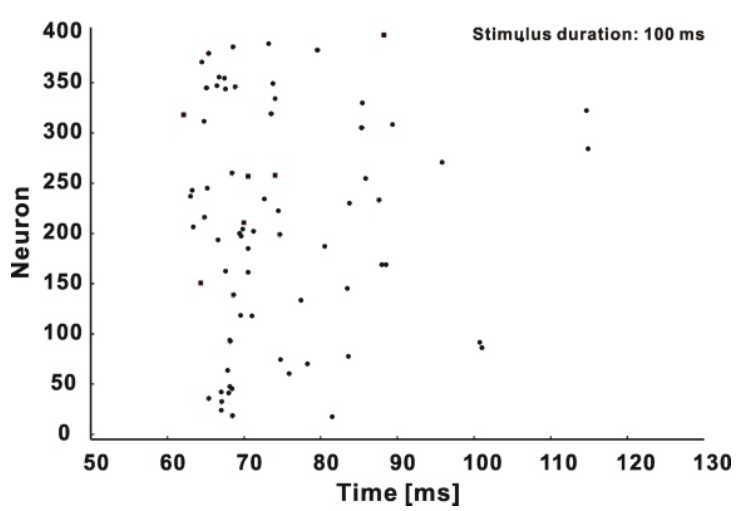

Figure 6. Raster plots of the large-scale neural network in response to stimuli with different durations. The configuration of the model is identical for Figure A to F. 

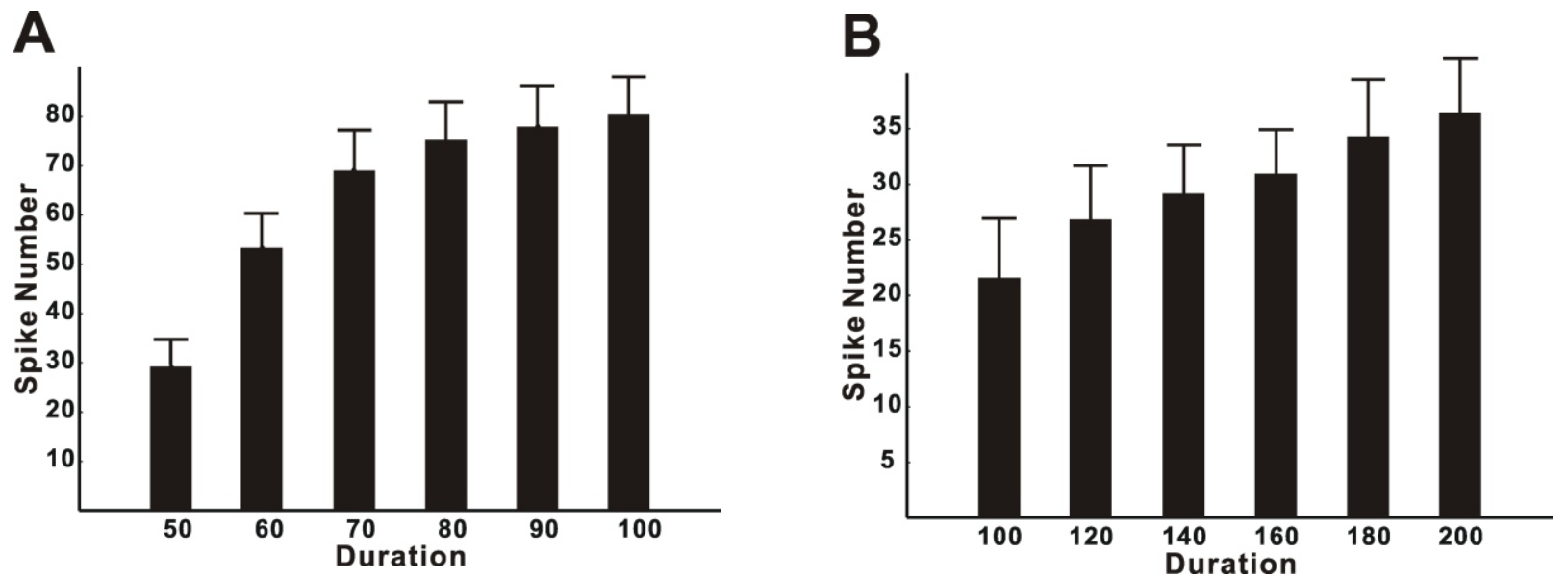

Figure 7. Recruitment of neuronal activities (activated numbers) for the large-scale model in response to stimuli with durations ranging from 50 to $100 \mathrm{~ms}$ (A, step $10 \mathrm{~ms}$ ) and 100 to $200 \mathrm{~ms}$ (B, step $20 \mathrm{~ms}$ ). The mean values of synaptic strength from input to excitatory neurons are 0.055 and 0.038 for results in Figure $A$ and $B$, respectively. Data are analyzed from 10 independent trials in the form of (Mean \pm S.D.).

activities are limited with in a narrow temporal window in absence of electrical coupling (A \& C).

The firing patterns of the large-scale model in response to stimuli with various durations are further tested. Stimuli with durations varying from $50 \mathrm{~ms}$ to $100 \mathrm{~ms}$ are applied to the network, with steps being 10 ms. Raster plots of typical spike activities of the network are given in Figure $\mathbf{6}, \mathbf{A}$ to $\mathbf{F}$. It is revealed that the model neurons fire in a sequential pattern, with more neurons being sequentially recruited in response to longer duration. Such recruitment process in response to durations ranging from $50 \mathrm{~ms}$ to $100 \mathrm{~ms}$ is averaged based on ten independent trials and the result is shown in Figure 7A.

Stimuli with durations varying from $50 \mathrm{~ms}$ to 100 $m s$ are applied and relevant results are given in Figure 6 and Figure 7A. However, models with this structure can effectively represent durations in other ranges while relevant parameters are changed. These parameters include the capacitance value of the I-F neuronal model, the time constant for chemical synaptic strength, the synaptic strengths from input neuron to the network et al. Stimuli with durations ranging from $100 \mathrm{~ms}$ to $200 \mathrm{~ms}$ are applied to the network, in which the mean value of synaptic strength from input neuron to the neural network $\left(\mathrm{Cm}_{s e}\right)$ are changed (from $0.055 \mu \mathrm{S}$ to $0.038 \mu \mathrm{S}$ ). The performance of the model (averaged across ten independent trials) is plotted in Figure 7B.

\section{DISCUSSION}

Temporal information processing in neural system is critical for animal behavior. Neuroscientists have tried a lot in understanding the neural basis of relevant processes via both experimental [6-10] and computational approaches [19-24].

In the present study, the computational results demonstrate that electrical synapses could effectively contribute to the formation of a spatio- temporal firing pattern of neuronal ensembles while each neuron with in the ensemble fires within different time windows, and the spatio-temporal pattern of the neuronal activities is capable of representing stimulus duration in the form of sequential firing activities of the spatially distributed neurons.

The contribution of electrical synapses in the formation of spatio-temporal firing pattern is particularly examined in the present study. However, it is necessary to mention that other factors can also contribute to this process. For example, membrane capacitance of specific neurons can be variable because of variation in surface area as well as the membrane capacitance value per unit area [25-28]. These changes can function in parallel to electrical synapses in influencing the sequential firing patterns of neuronal ensembles.

Special role of electrical synapse is proposed in our models and there are also experimental clues which indicated possible roles of electrical synapse in temporal information processing. Data demonstrated that gap junction coupling within inferior olive mediated by connexin 36 could add 10-20 of precision to the fine temporal coordination of muscle firing during movement [13].

Neurons in the present work are modeled following the classic I-F neuron fashion without any specific properties for temporal information processing. These neurons can be tuned to response to any non-temporal properties of natural stimulus and thereby function for the corresponding behavioral tasks. For example, these neurons could be tone selective neuron which function for auditory behavior, or mechanosensory neurons which function for mechanosensation. While both electrical and chemical synapses are universal in the central nervous system, the model results suggest that both the spatial and temporal neuronal activities produced 
at the sensory layer of neural system could be processed together by sharing the same neural circuit. Temporal content of external stimulus could be read out from spike patterns of neuronal ensembles in the brain.

\section{ACKNOWLEDGEMENTS}

The authors would like to thank Dr. Shi-Yong Huang for helpful discussion. This work was supported by grants from the Hi-Tech Research and Development Program of China(No. 2006AA01Z125).

\section{REFERENCE}

[1]Buonomano DV\& Karmarkar UR. How do we tell time? Neuroscientist 2002, 8:42-51.

[2] Mauk MD \& Buonomano DV. The neural basis of temporal processing. Annu Rev Neurosci 2004, 27:307-340.

[3]Ivry RB \& Spencer RM. The neural representation of time. Curr Opin Neurobiol 2004, 14:225-232.

[4]deCharms RC \& Zador A.Neural representation and the cortical code. Annu Rev Neurosci 2000, 23:613-647.

[5]Dayan, P. \& Abbott, LF () Theoretical Neuroscience, MIT Press 2001.

[6]Casseday JH, Ehrlich D \& Covey E. Neural tuning for sound duration: role of inhibitory mechanisms in the inferior colliculus. Science 1994,264:847-850.

[7] Galazyuk AV \& Feng AS. Encoding of sound duration by neurons in the auditory cortex of the little brown bat, Myotis lucifugus. J Comp Physiol 1997, [A] 180:301-311.

[8]He J., Hashikawa T., Ojima H. \& Kinouchi Y. () Temporal integration and duration tuning in the dorsal zone of cat auditory cortex. JNeurosci 1997, 17:2615-2625.

[9]Ehrlich D, Casseday JH \& Covey E. Neural tuning to sound duration in the inferior colliculus of the big brown bat, Eptesicus fuscus. JNeurophysiol 1997, 77:2360-2372.

[10]Fremouw T, Faure PA, Casseday JH \& Covey E. Duration selectivity of neurons in the inferior colliculus of the big brown bat: tolerance to changes in sound level. J Neurophysiol 2005, 94:1869-1878.

[11]Connors BW \& Long MA. Electrical synapses in the mammalian brain. Annu Rev Neurosci 2004, 27:393-418.

[12]Sohl G., Maxeiner S. \& Willecke K. Expression and functions of neuronal gapjunctions. Nat Rev Neurosci 2005, 6:191-200.

[13]Placantonakis DG, Bukovsky AA, Zeng XH, Kiem HP \& Welsh JP. Fundamental role of inferior olive connexin 36 in muscle coherence during tremor. Proc Natl Acad Sci U S A 2004, 101:7164-7169.

[14]Beaulieu C., Kisvarday Z., Somogyi P., Cynader M. \& Cowey A. Quantitative distribution of GABA-immunopositive and immunonegative neurons and synapses in the monkey striate cortex (area 17). Cereb Cortex 1992, 2:295-309.

[15]Troyer TW \& Miller KD. Physiological gain leads to high ISI variability in a simple model of a cortical regular spiking cell Neural Comput 1997,9:971-983.

[16]Rall W. Distinguishing theoretical synaptic potentials computed for different soma-dendritic distributions of synaptic input. J Neurophysiol 1967, 30:1138-1168.

[17]Nowotny T., Rabinovich MI, Huerta R. \& Abarbanel HD. Decoding temporal information through slow lateral excitation in the olfactory system of insects. J Comput Neurosci 2003, 15:271-281.

[18]Kopell N. \& Ermentrout B. Chemical and electrical synapses perform complementary roles in the synchronization of interneuronal networks. Proc Natl Acad Sci U S A 2004, 01:15482-15487.

[19]Hooper SL, Buchman E. \& Hobbs KH. A computational role for slow conductances: single-neuron models that measure duration. Nat Neurosci2002, 5:552-556.

[20]Buonomano DV. Decoding temporal information: A model based on short-term synaptic plasticity. J Neurosci 2000 20:1129-1141.

[21]Nowotny T, Rabinovich MI \& Abarbanel HD. Spatial representation of temporal information through spike-timing- dependent plasticity. Phys Rev E Stat Nonlin Soft Matter Phys 2003, 68:011908.

[22]Buonomano DV \& Merzenich MM. Temporal information transformed into a spatial code by a neural network with realistic properties. Science 1995, 267:1028-1030.

[23] Mauk MD \& Donegan NH. A model of Pavlovian eyelid conditioning based on the synaptic organization of the cerebellum. Learn Mem 1997, 4:130-158.

[24]Medina JF, Garcia KS, Nores WL, Taylor NM \& Mauk MD. Timing mechanisms in the cerebellum: testing predictions of a large-scale computer simulation. J Neurosci 2000, 20:55165525 .

[25]Chitwood RA, Hubbard A. \& Jaffe DB. Passive electrotonic properties of rat hippocampal CA3 interneurones. J Physiol 1999, 515 (Pt 3):743-756.

[26]Gentet LJ, Stuart GJ \& Clements JD. Direct measurement of specific membrane capacitance in neurons. Biophys J 2000, 79:314-320.

[27]Major G., Larkman AU, Jonas P., Sakmann B. \& Jack JJ Detailed passive cable models of whole-cell recorded CA3 pyramidal neurons in rat hippocampal slices. J Neurosci 1994 14:4613-4638.

[28]Thurbon D., Luscher HR, Hofstetter T. \& Redman SJ. Passive electrical properties of ventral horn neurons in rat spinal cord slices. J Neurophysiol 1998, 80:2485-2502. 